\title{
Investigating and identifying Chlamydia psittaci in asymptomatic and symptomatic domestic dogs in middle province of Iran
}

\author{
M.M. Sarmeidani ${ }^{1}$, P. Keihani ${ }^{2}$, P. Rezaei ${ }^{1}$, H. Momtaz ${ }^{3}$, S.H. Heidari ${ }^{1}$ \\ ${ }^{1}$ Veterinary Medicine, ${ }^{2}$ Small Animal Internal Medicine, ${ }^{3}$ Microbiology, Veterinary Collage, Islamic Azad University, Shahre \\ Kord Branch, Shahre Kord, Iran
}

(Received July 1, 2017; Accepted August 20, 2017)

\begin{abstract}
C. psittaci is one of the dog's pathogen which can cause respiratory disorders in various hosts and human beings. Chlamydiae are obligatory interacellular bacteria which belong to Chlamydiales. Conjunctival and pharyngeal swabs were taken from 50 captive dogs presented at veterinary clinics of Isfahan and Shahrekord to determine the percentage of infection and prevalence of C. Psittaci in domestic dogs. Samples were collected during 2014 from a total of 7 different breeds of dog; 1-German shepherd 2-Terrier 3- Mixed Poodle 4-Doberman pinscher 5-Persian sheepdog 6- Siberian husky 7-Pekingese breeds were sampled. The molecular PCR method was used to detect this microorganism in captive dogs and C. psittaci was detected in $9(18 \%)$ of them.
\end{abstract}

Keywords: C.psittaci, Domestic dogs, Molecular PCR method Available online at http://www.vetmedmosul.org/ijvs

$$
\begin{aligned}
& \text { التحقق وتحديد الاصابة بالكلاميديا بستس في الكلاب المريضة والسليمة في المحافظات الوسطى من ايران } \\
& \text { مهدي مرادي سرمدين'، بايمان خلاني '، بوراي رازكي'، حسان ممتاز '، سبد حسنين حيدري' } \\
& \text { ' فرع الطب الباطني، ‘ فرع الطب الباطني للحيو انات الصغيرة، فرع الاحياء المجهرية، كلية الطب البيطري، جامعة ازاد الاسلامية } \\
& \text { فرع شاهكرد، شاهكرد، ايران }
\end{aligned}
$$

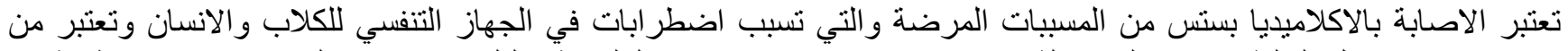

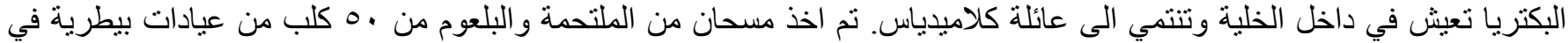

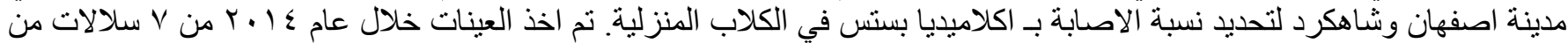

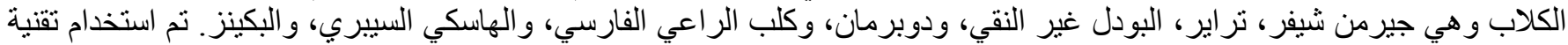

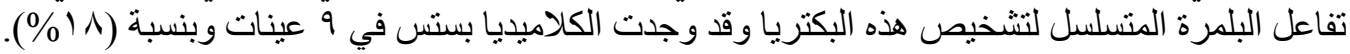

\section{Introduction}

C.psittaci is a compatible intracellular gram-negative Microorganism (M.o) that cause Chlamydiosis in canines. These M.o are capable of a unique developmental cycle which their multiplication is done by dual slit with cytoplasmic vacuoles connected to the host cell's membrane. It includes both forms of intercellular (Elementary body) and intracellular (Reticulate body). (1)
This infection can occur both with and without clinical symptoms. The most important clinical symptoms that arise subsequently due to chlamydial infection are: conjunctivitis, joint involvement, either individually or as polyarthritis, fever, anorexia, pneumonia, muscle and joint pain, inflammation of the tricuspid valve, moveable limp, keratitis and encephalitis. The infected way of disease is generally through direct contact with the wound and 
subsequently by scarring and chronic infection, possibility of diseases like osteomyelitis as well (1).

The pathological signs of infected dogs which can be mentioned are focal hepatitis, hyperplasia of the spleen, meningitis and fibrin - purulent polyarthritis (2).

Chlamydiosis can be acute, subacute and chronic. The dominant feature is conjunctivitis. The clinical symptoms of infecting by this pathogen are; involvement of upper respiratory tract, fever, eye discharge and sneezing. The organism has the ability to be systemic after inoculation of the conjunctiva, and be colonized in various tissues such as the tonsils, lungs, liver, spleen, the gastrointestinal tract and kidneys (1).

Limited epidemiological studies have been conducted on dogs. These studies show that more than 50 percent of traced antibodies of Chlamydia have been detected in dogs without clinical symptoms and 61.9 percent of them have been isolated from infected dogs (3). According to epidemiological reports and more important than that, this disease should receive considerable attention because of the contagiousness nature of the diseases among dogs and human. Investigating the percentage of infection of the pathogen factor is very important due to a common pathogen among humans, dogs, cats and pet birds such as (African greys, Parakeets and budgies) of doubles (3).

In this study, the samples collected from dogs with clinical symptoms (symptomatic) and without clinical symptoms referred to a veterinary clinic in Isfahan and Shahrekord and sent to the Department of Microbiology of Islamic Azad University of Shahrekord, they were investigated by using PCR and direct smear (Giemsa staining) method.

\section{Material and methods}

\section{Sampling}

Two different samples were taken from 50 dogs of different races, which are as follows: Direct smear of the conjunctiva of the eye was fixed by using a sterile swab on glass slides for staining (Giemsa) and then transferred into the test tube containing normal saline solution to conduct conventional molecular polymerase chain reaction (PCR) method. Direct samples was taken from the throat by using a sterile swab and then transferred into the test tube containing normal saline solution to conduct PCR method.

For the further investigations these samples were sent to the Department of Microbiology of Islamic Azad University of Shahrekord.

\section{Extracting DNA}

Normal salin solution-treated swabs were extracted using DNA extraction kit made by Sinagen Company of Iran according to DNA kit genomic. The sequence of the primers used in this research study conducted by Sykes et al
(5) which leads to the production of a piece with a length of $1094 \mathrm{bp}$ of ompA gene of C.psittaci was chosen to complete the following sequence: for the forward primer F: ATGAAAAAACTCTTGAAATCGG. The reserve primer has the sequence R: CAAGATTTTCTAGACTTCATTTTGTT.

The reaction of the PCR reaction in a final volume of 25 $\mu 1$ includes: $2.5 \mu \mathrm{l}$ of DNA template, $0.2 \mu \mathrm{m}$ of each primer, $200 \mu \mathrm{m}$ of dNTp mix (Fermntas Germany), 1.5 $\mathrm{MgCl}_{2} \mu \mathrm{m}$ and $2.5 \mu \mathrm{l}$ buffer of PCR and 1 unit of enzyme of Taq DNA Polymerase (Fermntas Germany) (5).

The samples were immediately put in a thermocycler and a temperature program was set as follows:

A cycle of $95^{\circ \mathrm{C}}$ for 5 minutes, 30 cycles of repetitive temperature $94^{\circ \mathrm{C}}$ for 1 minute and $72^{\circ \mathrm{C}}$ for 1 minute and a final round of 72 degrees for 8 minutes (5).

For carrying out the PCR of DNA extracted from the sample in direct testing (Giemsa staining) chlamydial bodies were used for mass unit as a positive control sample of distilled water was used as a negative control sample. At the end, the PCR product of tested samples on $1 \%$ agarose gel containing ethidium bromide in the presence of marker $1 \mathrm{~kb}$ base DNA (Fermntas Germany) was observed and recorded in a constant voltage of $90 \mathrm{~V}$ for $45 \mathrm{~min}$ and a UV light.

\section{Results}

The study results of 50 swab samples (a swab of conjunctiva and a swab of end of the throat) were taken from symptomatic (24 samples) and Asymptomatic (26 samples) dogs referred to pets clinic of Islamic Azad University of Shahrekord in Isfahan and Shahrekord and two dog shelters of Isfahan were carried out in two methods; Molecular PCR method and Giemsa staining:

\section{Molecular epidemiological findings}

In the PCR method, 9 samples (18\%) out of 50 samples had C.psittaci genome.

Dog samples were studied in three age groups; under 2 years (21), 2-4 years (23 samples) and more than 4 years (6 samples), in which the lowest infection rate was determined in dogs younger than 2 years $(9.52 \%)$ and the highest rate was in the group of 2-4 years old $(26.08 \%)$. Statistical analysis showed that there was no significant statistical difference between these three groups in Isfahan and Shahrekord $(\mathrm{P}<0.05)$.

Of the 50 samples, 27 samples were male and 23 were female. The infection rate of male samples was $11.11 \%$ and it was $26.08 \%$ for female dogs, but there was no significant difference between the infection rate and dog genders.

Twenty four samples of investigated dogs were with clinical symptoms, and 26 samples were without clinical symptoms. After taking the PCR test, 6 positive PCR (25\%) 
out of 24 samples with clinical symptoms, and 18 cases with negative PCR (72\%) out of 26 asymptomatic cases, 4 positive PCR $(15.3 \%), 22$ cases were negative PCR $(84.6 \%)$ were recorded. Statistical analysis of the results showed that there was no significant difference between the infection rate in symptomatic and asymptomatic dogs.

Seven breeds and 50 samples of dogs were studied in this research; 11 samples were German shepherd, 2 samples were Siberian husky, 12 were Iranian sheepdog, 17 were Pekingese, 2 samples were terrier, 4 samples were poodle and 2 samples were Doberman pinscher.

There was no significant difference between infection rate and C.psittaci between the various breeds that were studied $(\mathrm{P}<0.05)$.

Fifty samples taken from dogs were tested for the M.o by optical microscopy (Giemsa staining) in order to assess the accuracy and sensitivity of PCR test in the detection of C.psittaci of the dogs in this study. All the positive samples of PCR were also positive in Giemsa staining but 2 negative samples in microscopic methods were determined as infected by PCR method. Thus, the agreement of two tests for determining the infection with Chlamydia Psittaci is determined as the test that indicates the high sensitivity of PCR test in detecting the bacteria.

\section{Discussion}

C.psittaci is one of the disease factors in canines, birds (ornamental birds and other birds), various animals and humans, which are zoonotic and can, infect dogs and different hosts (causing respiratory problems, eye, etc.) is important. The disease is transmitted between multiple hosts directly and often by direct contact with nasal discharge, ocular discharge, respiratory tract secretions and feces of the infected host.

Nowadays, identifying C.psittaci is one of the important measures to control the disease in terms of public health and its spread to other hosts due to human willingness to keep dogs.

In this study, dogs from 7 different breeds were studied in the city of Isfahan and Shahrekord by using sensitive and accurate PCR test to identify OmpA C.psittaci genes in dogs and the results showed that $18 \%$ of the dogs, regardless of clinical symptoms related to this disease, were positive in C.psittaci. The important point is that so far there has been no study about investigating the C.psittaci infection in a dog in Iran.However, due to the survival and the probability of transmission of the M.o through carrier dogs it was required to determine the conditions of apparently healthy dogs (asymptomatic) infected with Chlamydia Psittaci and their role as popular domestic dogs in the spread among the human population, especially children and the dissemination of M.o between other breeds of dogs.
In present study in two cities of Isfahan and Shahrekord from 50 collected samples, 9 samples were positive and the highest infection rate was belonged to the German shepherd breed. According to the few clinical reports of the presence of C.psittaci in domestic dogs and especially various breeds of dogs, in some parts of Iran, it seems that there is a serious and important infection with this pathogen among dogs.

Recently some studies have been carried out in other parts of the world:

Holst et al. (2) study in 2010 investigated the C.psittaci in Swedish dogs. Real time-PCR test on samples taken from the conjunctival mucosa of the mouth and throat, rectum and genital tract of 52 dogs showed that 27 dogs were healthy and 25 dogs had clinical signs, including genital inflammation and conjunctiva symptoms and none of these dogs were infected with C.psittaci. Thus, it does not seem that Chlamydia has a big problem in conjunctiva and genital diseases of Swedish dogs.

Lambrechts et al. (3) in a study examined the role of chlamydia in bridging septic arthritis. After induction of chlamydia, the dog suffered from fever, swollen lymph nodes and imperfection in the joints. After separating the liquid of involved joints and providing smear, the samples were examined by light microscopy. The lipopolysaccharide antigen group A was observed in this test. Also the indirect fluorescent antibody test on serum was positive but complement fixation test was negative. Besides the test to culture the organism on egg yolk was inconclusive. In the end, the origin of induced chlamydial infection in the dog was not traced. Sprague et al. (4) investigated the formation of genotype $\mathrm{C}$ Chlamydia infection in four bitches with reversible Keratoconjunctivitis signs, respiratory distress and abortion that took place in a dog breeding center in Germany. After cell culture and immune-fluorescence examination of the conjunctiva, nasal and pharyngeal results proved the existence of Chlamydia Psittaci. Tian et al. (6) in another study investigated the serological and risk factors of chlamydia infection in dogs of southeast China. Indirect haemagglutination test on 591 samples taken from police dogs and stray dogs showed that the highest percentage of chlamydial infection belonged to stray dogs. The results showed the high prevalence of chlamydial infection in Yunnan province of China and the contagious potential for chlamydial infection is high. Therefore, special attention should be paid to the diagnosis of this disease in dogs.

Wu et al. (7) conducted a study in 2013 and determined the C.psittaci in domestic dogs and cats in Lanzhou, China. IHA tests done on blood samples from 121 domestic cats and 264 dogs showed that 13 cats and 32 dogs were infected with Chlamydia Psittaci. 


\section{Conclusion}

However, our study shows that although the rate of C.psittaci in asymptomatic dogs is low (18.18\%), but asymptomatic dogs can be considered as reservoirs of Chlamydia Psittaci. Due to detecting this M.o in taken samples from dogs older than 2 years and the absence of clinical symptoms in these dogs, it seems that the M.o can remain in the body for a long time and change the dog to a biological carrier and the infectious factor be excreted through feces and various secretions over time. Since these carriers are considered as potential risk for human and dogs infection therefore detecting, controlling and treating them will be very important. The purpose of this research was the importance of identifying this potential risk.

Given the fact that in the present study, samples were only taken from dogs (as a host) thus the findings of this study have big differences compared to other conducted studies on C.psittaci.

Since the German shepherd is the popular breed in the cities, the number of collected and positive samples of this breed compared to another breeds were high.However, the report of C.psittaci infection in dogs in the city of Isfahan and Shahrekord indicate the presence and dispersion of disease-causing organisms in different dogs.
The final purpose of this study was identifying the percentage of infection of C.psittaci in two cities of Isfahan and ShahreKord and giving suggestions to control and prevent the factor due to the zoonotic agent.

\section{References}

1. Greene CE. Infectious disease of the dog and cat. W.B. Saunders Co. Philadelphia, PA. 2012;pp: 362-370.

2. Holst BS, Hanas S, Bolske G, Forsberg CL. An investigation on the occurrence of chlamydiacea in Swedish dogs. Acta Vet Scandinavica. 2010;52:63

3. Lambrechts N, Picard J, Tustin RC. Chlamidia-induced septic polyarthritis in a dog. Tydskr S Afr Vet. 1999;70(1):40-42.

4. Sprague LD, Schubert E, Hotzel H, Scharf S, Sachse K. 2009.The detection of Chlamydia psittaci genotype $\mathrm{C}$ infection in dogs. Vet $\mathrm{J}$. 1999;181:274-279.

5. Sykes JE, Allen JL, Studdert VP, Browning GF. Detection of feline calicivirus, feline herpesvirus 1 and Chlamydia psittaci mucosal swans by multiplex RT-PCR/PCR. Vet Microbial. 2001;81(2):95-108.

6. Tian YM, Cao JF, Zhou DH, Zou FC, Miao Q, Liu ZL, Li BF, Lv RQ. $\mathrm{Du} \mathrm{XP}, \mathrm{Zhu} \mathrm{XQ}$. Seroprevalence and risk factor of Chlamydia infection in dogs in southwestern China. J Acta Tropica. 2014;130:6770.

7. Wu S M, Huang SY, Xu MJ, Zhou DH, Song HQ, Zhu XQ. Chlamydia felis exposure in companion dogs and cats in Lanzhou,China:a public health concern. BMC Vet Res. 2013;9:104. 